\title{
GcvR interacts with GcvA to inhibit activation of the Escherichia coli glycine cleavage operon
}

\author{
Angela C. Ghrist, Gary Heil and George V. Stauffer \\ Author for correspondence: George V. Stauffer. Tel: +1 319335 7791. Fax: +1 3193359006. \\ e-mail : george-stauffer@uiowa.edu
}

Department of Microbiology, The University of lowa, lowa City, IA 52242, USA

\begin{abstract}
The Escherichia coli glycine cleavage enzyme system, encoded by the gcvTHP operon, catalyses the oxidative cleavage of glycine to $\mathrm{CO}_{2}, \mathbf{N H}_{3}$ and a onecarbon methylene group. Transcription of the gcv operon is positively regulated by GcvA and negatively regulated by GcvA and GcvR. Using a LexAbased system for analysing protein heterodimerization, it is shown that GcvR interacts directly with GcvA in vivo to repress gcvTHP expression. Several mutations in either gcvA or gcv $R$ that result in a loss of $g c v$ repression also result in a loss of GcvA/GcvR heterodimerization. Finally, it is shown that the C-terminal half of GcvA is involved in its interaction with GcvR, whilst the entire GcvR protein appears to be necessary for heterodimerization.
\end{abstract}

Keywords: gcvTHP, repressor, activator, repression, antiactivation

\section{INTRODUCTION}

The Escherichia coli glycine cleavage enzyme system, encoded by the $g c v$ THP $(g c v)$ operon, catalyses the oxidative cleavage of glycine to $\mathrm{CO}_{2}, \mathrm{NH}_{3}$ and a onecarbon methylene group (Kikuchi, 1973; Meedel \& Pizer, 1974). This one-carbon group is transferred to the acceptor tetrahydrofolate to form 5,10-methylenetetrahydrofolate, a methyl donor used in the biosynthesis of purines, thymine, methionine and numerous methylated products (Mudd \& Cantoni, 1964). Transcription of the $g c v$ operon is activated by the GcvA protein in the presence of excess glycine. When glycine is limiting, GcvA actively represses expression of the operon; this repression is more severe in the presence of excess purines (Wilson et al., 1993a, b). GcvA, a member of the LysR family of transcriptional regulators, binds by way of a helix-turn-helix motif to three sites upstream of the gcv promoter (Wilson et al., 1995; Jourdan \& Stauffer, 1998) (Fig. 1). Binding sites 2 and 3 are required for activation of the operon in response to glycine, whereas all three sites are required for repression in response to purines (Wilson et al., 1995; Wonderling et al., 2000).

GcvA-mediated regulation of $g c v$ expression requires two additional proteins, Lrp and GcvR. Lrp, a global transcriptional regulator of genes involved in amino acid metabolism (Lin et al., 1992; Calvo \& Matthews, 1994), is required for both activation and repression of $g c v$ expression (Stauffer \& Stauffer, 1994). It has been

Abbreviations: AP, ampicillin; DBD, DNA-binding domain; gcv, gcvTHP; TC, tetracycline; TPEG, phenylethyl- $\beta$-D-thiogalactoside. proposed that Lrp plays a structural role in $g c v$ regulation, binding to and bending the DNA between GcvA-binding sites 1 and 2 to facilitate the formation of appropriate regulatory complexes (Stauffer \& Stauffer, 1999) (Fig. 1).

GcvR, a negative-acting protein required for GcvAmediated repression of $g c v$ transcription (Ghrist \& Stauffer, 1995), does not appear to regulate gcv expression directly, as it fails to bind to the $g c v$ control region in gel mobility shift assays (A. Ghrist, unpublished results). In addition, GcvR does not regulate gcv expression indirectly by regulating the expression of gcvA or gcvR (Ghrist \& Stauffer, 1998). Rather, repression of the $g c v$ operon depends on the relative levels of expression of $g c v A$ and $g c v R$; overexpression of $g c v A$ results in $g c v$ activation, whereas overexpression of $g c v R$ results in super-repression (Ghrist \& Stauffer, 1995). In light of these observations, we hypothesized that GcvR interacts directly with GcvA to control activation and repression of the $g c v$ operon (Ghrist \& Stauffer, 1998). In this study, we present evidence that supports this hypothesis. Using a LexA-based system for analysing protein heterodimerization, we show that GcvA and GcvR interact in vivo. In addition, we show that $g c v A$ and $g c v R$ mutations which decrease $g c v$ repression also decrease heterodimerization.

\section{METHODS}

Bacterial strains and plasmids. All strains used in this study were constructed in our laboratory and are derivatives of the E. coli K-12 strain GS162 [pheA905 thi $\Delta(\operatorname{argF}-\mathrm{lac}) U 169$ araD129 rpsL150 relA1 deoC1 flbB5301 ptsF25 rbsR]. Ad- 


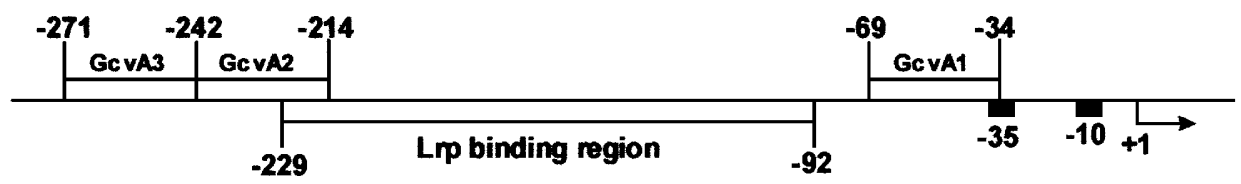

Fig. 1. gcv control region. The GcvA upstream (sites 3 and 2) and downstream (site 1) binding sites (Wilson et al., 1995) and the Lrp-binding region (Stauffer \& Stauffer, 1994) are shown relative to the gcv promoter $-35,-10$ and +1 sequences (Stauffer et al., 1993). Not to scale.

ditional strain designations and mutations are as follows: GS1111, $\Delta g c v R$ bcp $\Sigma$ neo; GS1118, $\Delta g c v$ A orf2 $2 a a d A ;$ GS1131, $\Delta g c v R$ bcp $\Sigma$ neo, $\Delta g c v A$ orf $2 \Sigma a a d A$. All lysogens denoted with $\lambda g c v T:$ : lac Z carry a single chromosomal copy of a $g c v T:: l a c Z$ reporter fusion in phage igt2 (Stauffer et al., 1993). The lysogen GS1131 sulA::lacZ carries a single chromosomal copy of the sulA::lacZ reporter fusion from strain SU202 (Dmitrova et al., 1998).

The multi-copy plasmid used in the $g c v R$ mutant screen was pBR322 (Bolivar et al., 1977). The low-copy-number plasmid used in this study was pSC101 (Cohen \& Chang, 1977) and the single-copy plasmid used was pGS311 (Ghrist \& Stauffer, 1995).

Media and growth conditions. The rich medium used was Luria-Bertani broth (LB) (Miller, 1992). The minimal media used were minimal salts (Vogel \& Bonner, 1956) supplemented with phenylalanine $\left(50 \mu \mathrm{g} \mathrm{ml}^{-1}\right)$, thiamin $\left(1 \mu \mathrm{g} \mathrm{ml} \mathrm{m}^{-1}\right)$, and either $0.4 \%$ glucose (GM) or $0.4 \%$ lactose (LM). Agar was added at $1.5 \%$ to make solid media. Additional supplements were added, where indicated, at the following concentrations : inosine, $50 \mu \mathrm{g} \mathrm{ml}^{-1}$; glycine, $300 \mu \mathrm{g} \mathrm{ml}^{-1}$; phenylethyl- $\beta$-Dthiogalactoside (TPEG), $2 \mathrm{mM}$; ampicillin (AP), $150 \mu \mathrm{g} \mathrm{ml}^{-1}$ for multi-copy plasmids and $30 \mu \mathrm{g} \mathrm{ml}^{-1}$ for single-copy plasmids; tetracycline (TC), $10 \mu \mathrm{g} \mathrm{ml}^{-1}$. $\lambda g c v T$ : : lacZ lysogens carry the $c \mathrm{I} 857$ mutation resulting in a temperature-sensitive repressor and were grown at $30^{\circ} \mathrm{C}$. All other strains were grown at $37^{\circ} \mathrm{C}$.

Construction of LexA fusion plasmids. Wild-type and mutant alleles of gcvA were cloned into pMS604 (Dmitrova et al., 1998) on PCR-generated BstEII-XhoI fragments, creating inframe fusions with the LexA DNA-binding domain (DBD). Wild-type and mutant $g c v R$ alleles were cloned into pDP804 (Dmitrova et al., 1998) on PCR-generated BssHII-BglII fragments, creating in-frame fusions with the LexA408 DBD variant. The nucleotide sequence of each PCR-generated fragment was verified by DNA sequence analysis.

$\boldsymbol{\beta}$-Galactosidase assays. $\beta$-Galactosidase assays were performed as described by Miller (1992). Each experiment was repeated at least twice, with each sample assayed in triplicate.

\section{RESULTS}

\section{GcvR interacts with GcvA in vivo}

To determine if GcvR interacts with GcvA in vivo, we used a LexA-based system for analysing protein heterodimerization developed for use in E. coli (Dmitrova et al., 1998). In this system, the reporter strain carries a $\lambda$ sulA::lacZ fusion under the control of a hybrid LexA operator containing a wild-type half-site and a mutant half-site (Fig. 2). The wild-type half-site is recognized by the wild-type LexA DBD and the mutant half-site is recognized by the LexA408 DBD variant. Interaction between protein domains fused to these LexA DBDs will create LexA/LexA408 heterodimers capable of repressing the $\lambda$ sulA::lacZ reporter (Fig. 2). For this experiment, $g c v A$ was fused downstream of the wildtype LexA DBD sequence of pMS604 and $g c v R$ was fused downstream of the LexA408 DBD sequence of pDP804. The expression of both fusion proteins is under the control of a lacUV5 promoter with a down mutation in the ribosome-binding site. Since the reporter strain GS1131 isulA : : lacZ carries a lacI deletion, both fusion proteins are constitutively expressed in this strain. The reporter strain transformed with one or both of these fusion plasmids was grown in LB (plus appropriate antibiotics) and $\beta$-galactosidase activity was determined. Expression of either fusion protein alone resulted in a small decrease in $\beta$-galactosidase activity (less than $30 \%$ ) (Table 1). However, co-expression of both fusion proteins resulted in a significant decrease in $\beta$-galactosidase activity (Table 1 , row 4). Because plasmids pMS604 and pDP804 are high-copy number and since each fusion protein alone had a small effect on sulA:: lac $Z$ expression, we tested whether the effects of the GcvA and GcvR fusion proteins alone were additive and partly responsible for some of the reduced expression of the sulA::lacZ fusion in the co-expression experiment. The wild-type $g c v A$ gene fused to the LexA DBD in pMS604 was cloned into the low-copy-number plasmid pSC101. Expression of the fusion protein from the low-copy-number plasmid did not cause a decrease in $\beta$-galactosidase activity (Table 1 , compare rows 1 and $10)$. When co-expressed with the GcvR fusion protein, there was still a significant reduction in $\beta$-galactosidase levels (Table 1, compare rows 1 and 11). These results indicate that GcvA and GcvR interact to form heterodimers in vivo.

\section{Isolation of $g c v R$ mutants unable to properly repress the gcv operon}

To determine if $g c v R$ mutants unable to properly repress $g c v$ expression might also be defective in their ability to interact with GcvA, the following strategy was used to isolate $g c v R$ mutants impaired in their ability to negatively regulate the $g c v$ operon. The wild-type lysogen GS162igcvT::lacZ, which carries a chromosomal $g c v T:: l a c Z$ reporter fusion, was transformed with a multi-copy plasmid pool carrying PCR-induced random base pair changes in $g c v R$. Transformants were selected on $\mathrm{LB}+\mathrm{AP}$ plates and then transferred to $\mathrm{LM}+\mathrm{AP}$ plates supplemented with the purine nucleoside inosine and TPEG (an inhibitor of $\beta$-galactosidase); this medium results in maximal repression of 


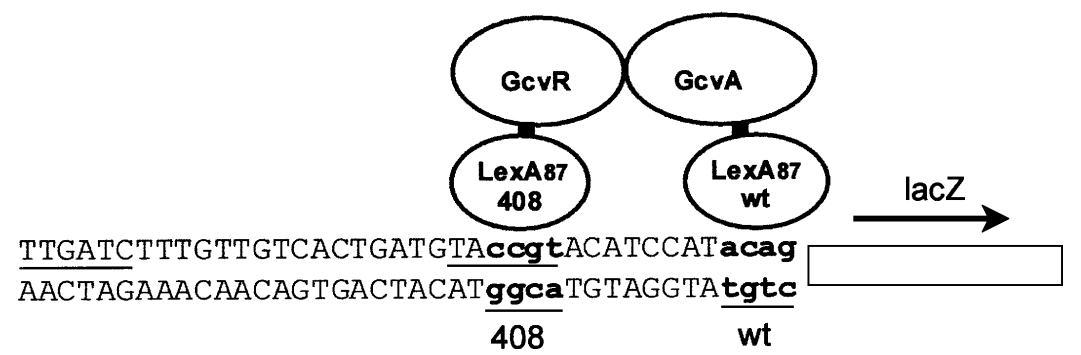

Fig. 2. Transcriptional repression by LexA heterodimer formation of GcvA and GcvR. The sulA -35 and -10 promoter elements are indicated by a line between the sequences and the mutant 408 and wildtype (wt) operator half-sites are in lower case (Dmitrova et al., 1998).

Table 1. Heterodimerization of GcvA and GcvR and their mutants

\begin{tabular}{|c|c|c|}
\hline$g c v A$ allele* & $g c v R$ allele $\dagger$ & $\begin{array}{c}\beta \text {-Galactosidase } \\
\text { activity } \ddagger\end{array}$ \\
\hline None & None & $1133 \pm 145$ \\
\hline None & $g c v R$ & $992 \pm 34$ \\
\hline$g c v A$ & None & $930 \pm 5$ \\
\hline$g c v A$ & $g c v R$ & $109 \pm 14$ \\
\hline$g c v A$ & $g c v R R 129 \mathrm{H}$ & $548 \pm 3$ \\
\hline$g c v A$ & $g c v R Q 144 \mathrm{P}$ & $484 \pm 20$ \\
\hline gcvAC169R & $g c v R$ & $286 \pm 46$ \\
\hline gcvAR197G & $g c v R$ & $493 \pm 23$ \\
\hline$g c v A \Delta 292-305$ & $g c v R$ & $687 \pm 16$ \\
\hline$g c v A \mathbb{S}$ & None & $1157 \pm 71$ \\
\hline$g c v A \mathbb{S}$ & $g c v R$ & $176 \pm 40$ \\
\hline
\end{tabular}

$* g c v A$ and its mutant alleles were fused in-frame to the lexA region of pMS604 (or pSC101\$).

$+g c v R$ and its mutant alleles were fused in-frame to the lexA408 region of $\mathrm{pDP} 804$.

‡GS1131 sulA::lacZ transformants were grown in LB plus appropriate antibiotics and assayed for $\beta$-galactosidase activity. Activity is expressed in Miller units (Miller, 1992).

the $g c v T:: l a c Z$ fusion and the inability to grow with lactose as the carbon source. Assuming that GcvR acts as a dimer (G. Heil, unpublished results), we reasoned that transformants expressing mutant GcvR proteins that could not interact with GcvA but that could still dimerize with wild-type GcvR and interfere with its ability to repress $g c v$ expression would be able to grow on the LM plates due to increased $\beta$-galactosidase activity. Five transformants with this phenotype were isolated. Plasmid DNA was purified from each of these transformants and used to transform the $\Delta g c v R$ strain GS1111igcvT: :lacZ. The resulting transformants were grown in GM supplemented with inosine and assayed for $\beta$-galactosidase activity. All five were found to have increased $\beta$-galactosidase activity as compared to GS1111igcvT::lacZ transformed with a multi-copy plasmid carrying the wild-type $g c v R$ allele, indicating that each encoded a GcvR protein defective in negative regulation of the $g c v$ operon (data not shown).

Each of the mutant $g c v R$ alleles was sequenced and the following mutations were identified: $g c v R \Delta 99-189$ and gcvR $\Delta 131-189$ contain stop codons at positions 99 and 131, respectively; $g c v R \Delta 106$ contains a single nucleotide
Table 2. $g c v A$ and $g c v R$ mutations and their effects on gcvT::lacZ expression

\begin{tabular}{|c|c|c|c|c|}
\hline \multirow[t]{2}{*}{ Strain* } & \multirow[t]{2}{*}{$\begin{array}{l}\text { Plasmid-encoded } g c v A \\
\text { or } g c v R \text { allele } \dagger\end{array}$} & \multicolumn{3}{|c|}{$\begin{array}{l}\beta \text {-Galactosidase activity } \\
\text { in cells grown in: } \mathbb{S}\end{array}$} \\
\hline & & GM & Glycine & Inosine \\
\hline Wild-type & None & 233 & 1395 & 22 \\
\hline$\Delta g c v R$ & None & 1756 & 2122 & 1209 \\
\hline$\Delta g c v R$ & $g c v R \Delta 131-189$ & 2041 & 2448 & 1340 \\
\hline$\Delta g c v R$ & $g c v R \Delta 106$ & 2070 & 2561 & 1435 \\
\hline$\Delta g c v R$ & gcvR $\Delta 99-189$ & 1863 & 2060 & 1111 \\
\hline$\Delta g c v R$ & gcvRR129H & 1156 & 1891 & 715 \\
\hline$\Delta g c v R$ & $g c v R Q 144 \mathrm{P}$ & 2011 & 1767 & 1362 \\
\hline$\Delta g c v A$ & None & 110 & 127 & 54 \\
\hline$\Delta g c v A$ & gcvAC169R & 529 & 1006 & 261 \\
\hline$\Delta g c v A$ & gcvAR197G & 1522 & 2373 & 1066 \\
\hline$\Delta g c v A$ & gcvA $\triangle 292-305$ & 1413 & 2026 & 1070 \\
\hline
\end{tabular}

*Transformants of GS162igcvT::lacZ (wild-type), GS1111$\lambda g c v T:: \operatorname{lac} Z(\Delta g c v R)$ or GS1118igcvT:: lacZ $(\Delta g c v A)$.

† All mutant alleles were expressed from the single-copy plasmid pGS311.

$\ddagger$ Activity is expressed in Miller units (Miller, 1992). All assays were repeated at least twice. Data from a representative experiment are shown.

\$Transformants were grown in GM+AP with supplements as indicated and assayed for $\beta$-galactosidase activity.

deletion (C) at codon 106, resulting in a reading frameshift and a stop codon at position 108, producing a protein that is 107 amino acids in size with two missense amino acids at its C-terminus; gcvRR129H contains an arginine to histidine change at position 129; and $g c v R Q 144 \mathrm{P}$ contains a glutamine to proline change at position 144 .

To determine the effects of these mutant alleles on $g c v$ expression when they are expressed at wild-type levels, each mutant $g c v R$ allele was cloned into the singlecopy plasmid pGS311. The resulting single-copy constructs were used to transform the $\Delta g c v R$ strain GS1111 lgcvT::lacZ. Transformants were grown in GM, GM + glycine and GM + inosine media and assayed for $\beta$-galactosidase activity (Table 2 ). The three deletion alleles as well as $g c v R Q 144 P$ led to a complete loss of $g c v$ repression in all three media, exhibiting $\beta$ galactosidase activities nearly identical to the levels observed in the $\Delta g c v$ R control. The $g c v R R 129 \mathrm{H}$ allele 


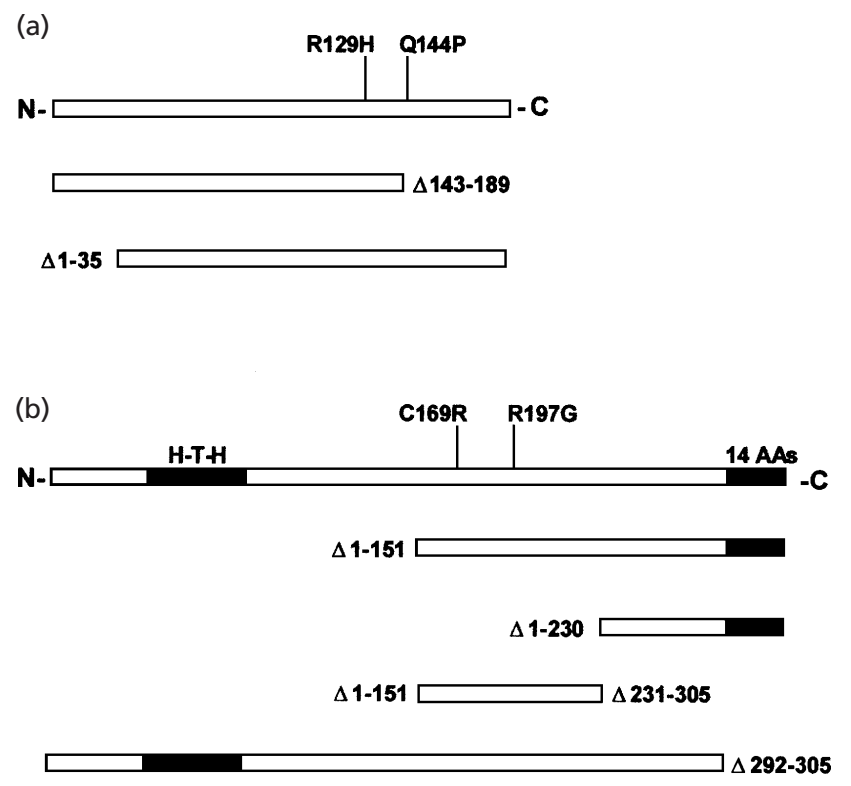

Fig. 3. (a) The 189 amino acid GcvR protein and the GcvR fragments fused to the LexA87 408 headpiece in plasmid pDP804 used to define regions of GcvR involved in interacting with GcvA. The R129 and Q144 amino acids involved in negative regulation are indicated relative to the deletion end points. (b) The 305 amino acid GcvA protein and the GcvA fragments fused to the LexA87 wild-type headpiece in plasmid pMS604 used to define the region of GcvA involved in interacting with GcvR. The C169 and R197 amino acids and the C-terminal 14 amino acids involved in negative regulation are indicated. H-T-H, helix-turn-helix domain. Not to scale.

also led to a loss of repression, but the phenotype was less severe. It should be noted that the 1.5-2-fold repression observed in GM+inosine medium is due to the PurR repressor, which has been shown to repress transcription of the $g c v$ operon independently of GcvA/ GcvR-mediated repression (Wilson et al., 1993a).

\section{GcvR mutants unable to repress gcv expression are impaired in their ability to interact with GcvA}

If GcvA interacts directly with GcvR to regulate the $g c v$ operon, one possible explanation for the loss of repression displayed by the $g c v R$ mutants described above is that they are unable to interact with GcvA. Therefore, the $g c v R \mathrm{Q} 144 \mathrm{P}$ and $g c v R \mathrm{R} 129 \mathrm{H}$ alleles were cloned into pDP804 and tested for their ability to interact with GcvA using the protein heterodimerization system. Both mutant alleles were impaired in their ability to interact with GcvA, displaying an approximately $4 \cdot 5-5 \cdot 5$-fold increase in expression of the $\lambda$ sulA::lacZ reporter fusion as compared to the wild-type $g c v R$ allele (Table 1, compare row 4 with rows 5 and 6 ).

\section{Deletion analysis of $g c v R$}

To define the region(s) of GcvR involved in its interaction with GcvA, a 3' deletion ( $g c v R \Delta 143-189)$ and a $5^{\prime}$ deletion $(g c v R \Delta 1-35)$ of $g c v R$ (Fig. 3a) were cloned
Table 3. Deletions of $g c v A$ and $g c v R$ and their effects on GcvA/GcvR interaction

\begin{tabular}{|llc|}
\hline $\boldsymbol{g} \boldsymbol{c} \boldsymbol{A}$ allele* & $\boldsymbol{g} \boldsymbol{c v} \boldsymbol{R}$ allele f $^{*}$ & $\begin{array}{c}\boldsymbol{\beta} \text {-Galactosidase } \\
\text { activity }\end{array}$ \\
\hline None & None & $1195 \pm 165$ \\
None & $g c v R$ & $1102 \pm 96$ \\
$g c v A$ & None & $999 \pm 79$ \\
$g c v A$ & $g c v R \Delta 143-189$ & $711 \pm 2$ \\
$g c v A$ & $g c v R \Delta 1-35$ & $949 \pm 129$ \\
$g c v A \Delta 1-151$ & $g c v R$ & $143 \pm 13$ \\
$g c v A \Delta 1-230$ & $g c v R$ & $821 \pm 18$ \\
$g c v A \Delta 1-151 \cdot \Delta 231-305$ & $g c v R$ & $1076 \pm 108$ \\
\hline
\end{tabular}

" $g c v A$ and its mutant alleles were fused in-frame to the $\operatorname{lex} A$ region of pMS604.

$\dagger g c v R$ and its mutant alleles were fused in-frame to the lexA 408 region of pDP804.

$\ddagger$ GS1131 sulA::lacZ transformants were grown in LB plus appropriate antibiotics and assayed for $\beta$-galactosidase activity. Activity is expressed in Miller units (Miller, 1992).

into pDP804 and tested for their ability to interact with GcvA using the LexA heterodimerization assay. Since all of the $g c v R$ mutations described above were located in the 3' half of $g c v R$, it was not surprising that deletion of the C-terminal 47 residues of GcvR, which includes the glutamine at position 144, resulted in a loss of interaction with GcvA (Table 3, row 4). It was somewhat surprising, however, that deletion of the N-terminal 35 residues also resulted in a loss of interaction with GcvA (Table 3, row 5).

\section{Isolation of GcvA mutants refractory to inhibition by GcvR}

In a previous study, two $g c v A$ mutants that are able to activate but not repress $g c v$ expression were isolated but not characterized (Jourdan \& Stauffer, 1998). In that same study, a deletion of the sequence encoding the Cterminal 14 amino acids of GcvA ( $\Delta g c v A 944$; renamed gcvA $\triangle 292-305$ in this study) (Fig. 3b) was constructed and shown to have the same phenotype. During this study, the uncharacterized gcvA mutant alleles were sequenced and the mutations were identified as a cysteine to arginine change at position 169 ( $g c v A C 169 \mathrm{R}$ ) and an arginine to glycine change at position 197 (gcvAR197G) (Fig. 3b). To measure the effects of these mutations on $g c v$ expression, they were cloned into the single-copy plasmid pGS311 and the resulting constructs were used to transform the $\Delta g c v A$ reporter strain GS1118igcvT::lacZ. Transformants were grown in GM, GM + glycine and GM + inosine media and assayed for $\beta$-galactosidase activity. As expected, the $\triangle g c v A$ control lysogen showed no significant change in $\beta$-galactosidase activity under any growth condition, except for the twofold PurR effect (Table 2). The gcvAR197G and $g c v A \Delta 292-305$ mutations resulted in a complete loss of $g c v$ repression, producing levels of $\beta$ - 
galactosidase activity similar to that observed in the $\Delta g c v R$ control (Table 2, rows 10 and 11). The gcvAC169R mutation also led to a loss of $g c v$ repression, although the phenotype was not as severe (Table 2, row 9).

\section{GcvA mutants unable to repress gcv expression are impaired in their ability to interact with GcvR}

To test whether the $g c v A$ mutants described above are defective in their ability to interact with GcvR, each mutant allele was cloned into pMS604 and its interaction with GcvR measured using the LexA heterodimerization system. The gcvAC169R mutation resulted in a small loss of interaction with GcvR, whilst the gcvAR197G and gcvA $\triangle 292-305$ mutations resulted in more severe losses of interaction with GcvR (Table 1, compare row 4 with rows 7, 8 and 9). These results are consistent with the effects of the mutations on repression of the $g c v$ operon.

\section{The C-terminal half of GcvA is able to interact with GcvR}

To define the region(s) of GcvA that is involved in its interaction with GcvR, several $5^{\prime}$ and $3^{\prime}$ deletions of $g c v A$ (gcvA $1-151, g c v A \Delta 1-230$ and $g c v A \Delta 1-$ 151- $\Delta 231-305$ ) (Fig. 3b) were cloned into pMS604 and tested for their ability to interact with GcvR using the protein heterodimerization system. Deletion of the $\mathrm{N}$ terminal 151 amino acids of GcvA had no effect on its ability to interact with GcvR (Table 3, row 6). However, deletion of the N-terminal 230 amino acids, which deletes the cysteine at position 169 and the arginine at position 197, or deletion of amino acids 1-151 and 231-305, resulted in significant loss of interaction with GcvR (Table 3, rows 7 and 8). These results suggest that the GcvA/GcvR interaction requires the C-terminal half of GcvA.

\section{The C-terminal half of GcvA interferes with inhibition of wild-type GcvA by GcvR}

Sequence and mutational analysis indicate that the Nterminal half of GcvA is involved in DNA binding and activation (Wilson \& Stauffer, 1994; Jourdan \& Stauffer, 1998) and results from this study suggest that the C-terminal half of GcvA is involved in an interaction with GcvR. To determine if overexpression of the C-terminal half of GcvA would lead to activation of the $g c v$ operon by interfering with the ability of GcvR to inhibit wild-type GcvA, the wild-type strain GS162igcvT::lacZ and the $\Delta g c v A$ strain GS1118igcvT::lacZ were transformed with plasmids carrying the lexA-gcvA$\Delta 1-151$ and $\operatorname{lex} A-g c v A \Delta 1-230$ alleles used in the heterodimerization experiment. Transformants were grown in GM, GM+glycine and GM+inosine media and assayed for $\beta$-galactosidase activity. Transformation of the $\triangle g c v A$ strain GS1118igcvT: : lacZ with either plasmid resulted in low non-inducible $g c v$ expression, identical to that observed
Table 4. LexA-GcvA $\Delta 1-151$ interferes with inhibition of wild-type GcvA by GcvR

\begin{tabular}{|lccc|}
\hline$g c v A$ allele* & \multicolumn{3}{c|}{$\begin{array}{c}\boldsymbol{\beta} \text {-Galactosidase activity } \\
\text { cells grown in }: \neq\end{array}$} \\
\cline { 2 - 4 } & GM & Glycine & Inosine \\
\hline None & 135 & 817 & 21 \\
gcvA $\Delta 1-151$ & 847 & 994 & 340 \\
gcvA $\Delta 1-230$ & 165 & 914 & 27 \\
\hline
\end{tabular}

*gcvA deletion alleles were fused to the lexA408 region of plasmid pMS604.

† Activity is expressed in Miller units (Miller, 1992). All assays were repeated at least twice. Data from a representative experiment are shown.

‡GS162 $\mathrm{gcv}$ T:: lacZ transformants were grown in GM+TC medium with supplements as indicated and assayed for $\beta$ galactosidase activity.

in the untransformed control (data not shown). Thus, neither LexA- $\Delta \mathrm{Gcv} A$ protein fusion is able to activate the gcvT::lacZ fusion. Transformation of wild-type strain GS162 $\lambda g c v T$ : : lacZ resulted in normal regulation of $g c v$ expression in the case of the lexA-gcvA $\Delta 1-230$ allele, whereas transformation with the $\operatorname{lexA-}$ gcvA $\Delta 1-151$ allele led to constitutive activation in all three media (Table 4).

\section{DISCUSSION}

In this report, we present evidence to support a model in which GcvR interacts with GcvA to prevent activation of the $g c v$ operon. Using a LexA-based system for measuring protein heterodimerization, we were able to show that GcvA and GcvR interact in vivo. In addition, mutations in both $g c v A$ and $g c v R$ which result in a loss of $g c v$ repression also result in a loss of GcvA/GcvR interaction. Thus, there is a strong correlation between the ability of these proteins to form heterodimers and their ability to repress expression of the $g c v$ operon.

Three lines of evidence suggest that the C-terminal half of GcvA is involved in heterodimerization with GcvR. First, substitution mutants of GcvA which result in a loss of interaction with GcvR lie in the C-terminal half of GcvA. Second, deletion of the N-terminal half of GcvA has no effect on its ability to interact with GcvR, whereas deletion of the C-terminal 14 amino acids results in a complete loss of heterodimerization. Finally, although unable to activate $g c v$ transcription, the Cterminal half of GcvA is able to interfere with GcvRmediated repression of wild-type GcvA, probably by titrating the available antiactivator, leading to constitutive activation of the operon (Table 4). Together, these results suggest that the C-terminal half of GcvA is involved in an interaction with GcvR.

All of the $g c v R$ mutations identified in this study that result in a decrease in repression of the $g c v$ operon as 
well as a decrease in heterodimerization lie in the Cterminal half of the protein, suggesting that this region is involved in an interaction with GcvA. Consistent with these results, a deletion of the C-terminal 47 amino acids resulted in a loss of interaction with GcvA (Table 3). However, deletion of the $\mathrm{N}$-terminal 35 amino acids also resulted in a loss of heterodimerization (Table 3). Since our screen relied on the ability of mutant GcvR to interact with wild-type GcvR, it is possible that mutations in the N-terminal region that would have resulted in a loss of GcvR interaction with GcvA were excluded from the screen as the mutations might have also resulted in a loss of GcvR homodimerization. It is also possible that deletion of this region of $g c v R$ results in a decrease in expression or protein stability.

While it is clear that GcvR interacts with GcvA to repress the $g c v$ operon, it is not clear how this interaction is modified by glycine and purines and, in turn, how this modification leads to the correct regulation of $g c v$ expression. In previous studies, we have shown that neither glycine nor purines have an effect on the expression of $g c v A$ or $g c v R$ (Wilson \& Stauffer, 1994; Ghrist \& Stauffer, 1998). These results led us to propose a model in which GcvA homocomplexes function as activators, whilst GcvA/GcvR heterocomplexes function as repressors. In this model, the coeffectors influence the type of complex formed; glycine promotes the formation of activation complexes, whilst purines promote the formation of repression complexes. Increasing the expression of GcvA or GcvR would force the formation of activation or repression complexes, respectively. We used the LexA-based heterodimerization system to measure the effects of glycine and inosine on the ability of GcvA and GcvR to interact by growing transformants in GM, GM + glycine and GM + inosine media prior to assaying for $\beta$-galactosidase activity. However, glycine and inosine had no significant effect on the GcvA/GcvR interaction (data not shown). This may be due to the high level of expression of both fusion proteins in this assay and in vitro studies with purified GcvA and GcvR will be needed to provide further data to prove or disprove this model.

Regulation of the $g c v$ operon by GcvA and GcvR is part of a growing number of prokaryotic regulatory systems involving an activator/antiactivator complex, including TraR/TraM regulation of conjugal transfer of the $\mathrm{Ti}$ plasmid in Agrobacterium tumefaciens (Hwang et al., 1999), NifA/NifL regulation of nitrogen fixation in Klebsiella pneumoniae (Narberhaus et al., 1995), ComK/MecA/ClpC regulation of competence in $\mathrm{Ba}$ cillus subtilis (Turgay et al., 1998) and CRP/CytR regulation of operons encoding the nucleoside transport and biosynthesis enzymes (Valentin-Hansen et al., 1996). In the case of TraR/TraM, NifA/NifL and ComK/ $\mathrm{MecA} / \mathrm{ClpC}$, the activator is sequestered by the antiactivator(s), thus preventing binding and activation. In the case of CRP/CytR, the antiactivator binds DNA between activator-binding sites, forming a nucleoprotein complex which prevents activation (ValentinHansen et al., 1996). Evidence suggests that GcvR could act in both ways to negatively regulate $g c v$ expression. As discussed above, the C-terminal half of GcvA, deleted for the DNA-binding region (Jourdan \& Stauffer, 1998), prevents GcvR-mediated repression. This suggests that GcvR may function by sequestering GcvA in an inactive form. On the other hand, overexpressing GcvR in a $g c v A^{+}$strain results in a lower level of $g c v$ expression than that observed in a $g c v A$ mutant (Ghrist \& Stauffer, 1995). In addition, GcvA/GcvR, in the presence of inosine, repress a $g c v T:$ : lac $Z$ fusion four- to fivefold in a GcvA-binding site 1-dependent manner (Wonderling et al., 2000). These results suggest that GcvA and GcvR act together at the $g c v$ control region to directly repress gcv expression.

\section{ACKNOWLEDGEMENTS}

We thank Michele Granger for generously providing pMS604, pDP804 and SU202.

\section{REFERENCES}

Bolivar, F., Rodriguez, R. L., Greene, P. J., Betlach, M. C., Heyneker, H. L., Boyer, H. W., Crosa, J. H. \& Falkow, S. (1977). Construction and characterization of new cloning vehicles. II. A multipurpose cloning system. Gene 2, 95-113.

Calvo, J. M. \& Matthews, R. G. (1994). The leucine-responsive regulatory protein, a global regulator of metabolism in Escherichia coli. Microbiol Rev 58, 466-490.

Cohen, S. C. \& Chang, A. C. Y. (1977). Revised interpretation of the origin of the pSC101 plasmid. J Bacteriol 132, 734-737.

Dmitrova, M., Younes-Cauet, G., Oertel-Buchheit, P., Porte, D., Schnarr, M. \& Granger-Schnarr, M. (1998). A new LexA-based genetic system for monitoring and analyzing protein heterodimerization in Escherichia coli. Mol Gen Genet 257, 205-212.

Ghrist, A. C. \& Stauffer, G. V. (1995). Characterization of the Escherichia coli $g c v R$ gene encoding a negative regulator of $g c v$ expression. J Bacteriol 177, 4980-4984.

Ghrist, A. C. \& Stauffer, G. V. (1998). Promoter characterization and constitutive expression of the Escherichia coli gcvR gene. J Bacteriol 180, 1803-1807.

Hwang, I., Smyth, A. J., Luo, Z.-Q. \& Farrand, S. K. (1999). Modulating quorum sensing by antiactivation: TraM interacts with TraR to inhibit activation of Ti plasmid conjugal transfer genes. Mol Microbiol 34, 282-294.

Jourdan, A. D. \& Stauffer, G. V. (1998). Mutational analysis of the transcriptional regulator GcvA: amino acids important for activation, repression, and DNA binding. J Bacteriol 180, $4865-4871$.

Kikuchi, G. (1973). The glycine cleavage system: composition, reaction mechanism, and physiological significance. Mol Cell Biochem 1, 169-187.

Lin, R., D'Ari, R. \& Newman, E. B. (1992). $\lambda$ placMu insertions in genes of the leucine regulon: extension of the regulon to genes not regulated by leucine. J Bacteriol 174, 1948-1955.

Meedel, T. H. \& Pizer, L. I. (1974). Regulation of one-carbon biosynthesis and utilization in Escherichia coli. J Bacteriol 118, 905-910.

Miller, J. H. (1992). A Short Course in Bacterial Genetics. Cold Spring Harbor, NY: Cold Spring Harbor Laboratory.

Mudd, S. H. \& Cantoni, G. L. (1964). Biological transmethylation, 
methyl-group neogenesis and other 'one-carbon' metabolic reactions dependent upon tetrahydrofolic acid. In Comprehensive Biochemistry, pp. 1-47. Edited by M. M. Florkin \& E. H. Stotz. Amsterdam: Elsevier.

Narberhaus, F., Lee, H.-S., Schmitz, R. A., He, L. \& Kustu, S. (1995). The C-terminal domain of NifL is sufficient to inhibit NifA activity. J Bacteriol 177, 5078-5087.

Stauffer, L. T. \& Stauffer, G. V. (1994). Characterization of the $g c v$ control region from Escherichia coli. J Bacteriol 176, 6159-6164.

Stauffer, L. T. \& Stauffer, G. V. (1999). Role for the leucineresponsive regulatory protein (Lrp) as a structural protein in regulating the Escherichia coli gcvTHP operon. Microbiology 145, 569-576.

Stauffer, L. T., Ghrist, A. \& Stauffer, G. V. (1993). The Escherichia coli gcvT gene encoding the T-protein of the glycine cleavage enzyme system. DNA Seq-J DNA Seq Mapping 3, 339-346.

Turgay, K., Hahn, J., Burghoorn, J. \& Dubnau, D. (1998). Competence in Bacillus subtilis is controlled by regulated proteolysis of a transcription factor. EMBO J 17, 6730-6738.

Valentin-Hansen, P., Sogaard-Andersen, L. \& Pedersen, H. (1996). A flexible partnership: the $c y t R$ anti-activator and the cAMPCRP activator protein, comrades in transcription control. Mol Microbiol 20, 461-466.
Vogel, H. J. \& Bonner, D. M. (1956). Acetylornithinase of Escherichia coli: partial purification and some properties. J Biol Chem 218, 97-106.

Wilson, R. L. \& Stauffer, G. V. (1994). DNA sequence and characterization of GcvA, a LysR family regulatory protein for the Escherichia coli glycine cleavage enzyme system. J Bacteriol 176, 2862-2868.

Wilson, R. L., Stauffer, L. T. \& Stauffer, G. V. (1993a). Roles of the GcvA and PurR proteins in negative regulation of the Escherichia coli glycine cleavage enzyme system. J Bacteriol 175, 5129-5134.

Wilson, R. L., Steiert, P. S. \& Stauffer, G. V. (1993b). Positive regulation of the Escherichia coli glycine cleavage enzyme system. J Bacteriol 175, 902-904.

Wilson, R. L., Urbanowski, M. L. \& Stauffer, G. V. (1995). DNA binding sites of the LysR-type regulator GcvA in the $g c v$ and $g c v A$ control regions of Escherichia coli. J Bacteriol 177, 4940-4946.

Wonderling, L. D., Urbanowski, M. L. \& Stauffer, G. V. (2000). GcvA binding site 1 in the gcvTHP promoter of Escherichia coli is required for GcvA-mediated repression but not for GcvAmediated activation. Microbiology 146, 2909-2918.

Received 29 December 2000; revised 23 March 2001; accepted 23 April 2001. 